\title{
Oscillations in the inflaton potential?
}

\author{
Cédric Pahud, ${ }^{1,2}$ Marc Kamionkowski, ${ }^{2}$ and Andrew R. Liddle ${ }^{1}$ \\ ${ }^{1}$ Astronomy Centre, University of Sussex, Brighton BN1 9QH, United Kingdom \\ ${ }^{2}$ California Institute of Technology, Mail Code 130-33, Pasadena, California 91125, USA
}

(Received 4 July 2008; published 3 April 2009)

\begin{abstract}
We consider a class of inflationary models with small oscillations imprinted on an otherwise smooth inflaton potential. These oscillations are manifest as oscillations in the power spectrum of primordial perturbations, which then give rise to oscillating departures from the standard cosmic microwave background power spectrum. We show that current data from the Wilkinson Microwave Anisotropy Probe constrain the amplitude of a sinusoidal variation in the inflaton potential to have an amplitude less than $3 \times 10^{-5}$. We anticipate that the smallest detectable such oscillations in Planck will be roughly an order of magnitude smaller, with slight improvements possible with a post-Planck cosmic-variance limited experiment.
\end{abstract}

DOI: 10.1103/PhysRevD.79.083503

PACS numbers: $98.80 .-\mathrm{k}$

\section{INTRODUCTION}

Cosmic microwave background (CMB) experiments continue to be consistent with the simplest predictions of inflationary models, even as the data become increasingly precise [1]. Constraints on the amplitude and spectral index of primordial density perturbations, and on the amplitude of the inflationary gravitational-wave background, can now be used to constrain the parameter space of the inflaton potential. In most of the current analyses the inflaton potential is parameterized in terms of its amplitude $V(\phi)$ and first and second derivatives, $V^{\prime}(\phi)$ and $V^{\prime \prime}(\phi)$.

Given the recent advances in data quality, as well as the improvements anticipated with forthcoming experiments (e.g., the Planck satellite [2], to be launched imminently), it is worth asking whether the data can be used to study more complicated forms of the potential. For example, Adams et al. [3] showed that supergravity-inspired models may give rise to inflaton potentials with a large number of steps. Each step corresponds to a symmetry-breaking phase transition in a field coupled to the inflaton. The inflaton mass then changes suddenly when each transition occurs. These steps are responsible for unusual inflaton dynamics, often represented as a hybrid inflation model [4], and they will create oscillating features in the primordial power spectrum. Oscillations can also be directly imprinted on the inflaton potential itself, due to some trans-Planckian physics [5-8]. Finally, some other mechanisms may create features in the primordial power spectrum [9]. There may also be empirical motivations to consider more complicated potentials, as several CMB analyses suggest that the CMB power spectrum may be better fit by primordial power spectra with features than by smooth power spectra [10-13].

In this paper, we consider a class of inflationary models that feature periodic oscillations imprinted on a smooth inflaton potential, which then give rise to oscillations in the primordial power spectrum. We look for these oscillations in the Wilkinson Microwave Anisotropy Probe (WMAP) data, and then determine the smallest oscillation amplitude that will be probed with forthcoming experiments. Our work is somewhat similar to that in Ref. [14], which considers oscillations in the primordial power spectrum that arise from a step in the inflaton potential, but differs in that we consider wiggles in the inflaton potential itself. There is also related work in Ref. [15], which considers oscillations in the CMB power spectrum from a rapid phase transition during inflation. However, the works most closely related to ours are Ref. [16], discussed further below, and Ref. [17]. The latter considers oscillations in the inflaton potential in natural inflation models; while they focused on constraints from existing data, we forecast also the detectability with future measurements.

The plan of this paper is as follows. Section II introduces the model and discusses the calculation of the power spectrum. Section III presents numerical results for the primordial, matter, and CMB power spectra. Section IV presents results of our search for oscillations in the WMAP data. Section V discusses the forecasts for the detectability of oscillations in future experiments; we consider here both Planck and a post-Planck cosmic-variance limited experiment. We summarize and provide some concluding remarks in Sec. VI.

\section{THE OSCILLATING MODEL}

In order to study the effects of small oscillations in the inflaton potential, we begin with a simple base inflationary model that is consistent with current data. We consider the simplest such model, namely, a quadratic inflaton potential, $V_{0}(\phi)=\frac{1}{2} m^{2} \phi^{2}$, with $m \simeq \sqrt{8 \pi} \times 10^{-6} M_{\mathrm{Pl}}$. This potential has a corresponding $\mathrm{CMB}$ amplitude $A_{s}=1.2 \times 10^{-9}$ for a $\Lambda \mathrm{CDM}$ model. Once normalized with the best-fit WMAP5 data, we get $n_{s}=0.96$ for a pivot scale $k_{\star}=0.002 \mathrm{Mpc}^{-1}$, consistent with current constraints [18]. 
We then superimpose on this smooth potential a sinusoidal fluctuation to give a potential of the form

$$
V(\phi)=\frac{1}{2} m^{2} \phi^{2}\left[1+\alpha \sin \left(\frac{\phi}{\beta M_{\mathrm{Pl}}}+\delta\right)\right],
$$

parameterized by an amplitude of oscillations and a parameter $\beta$ that characterizes the frequency. The amplitude $\alpha$ is assumed to be small so that the inflaton does not get stuck in one of the local minima introduced in the potential by the oscillations; we discuss the precise constraint later. We choose the phase $\delta=0$ and explain below why our results will be the same for different values.

Exactly this potential was studied recently in Ref. [16], but their focus was on predictions for non-Gaussianity rather than comparison of the power spectrum with observations. Like those authors, we adopt this potential on phenomenological grounds, though there may be ways of motivating it physically, e.g., along the lines of Ref. [19].

The homogeneous dynamics are dictated by the Friedmann equation for the scale factor and the inflaton equation of motion. The Friedmann equation (in units where $8 \pi G=c=\hbar=1$ ) for a universe containing a scalar field $\phi(t)$ with potential $V(\phi)$ is

$$
3 H^{2} \equiv 3\left(\frac{\dot{a}}{a}\right)^{2}=\frac{1}{2} \dot{\phi}^{2}+V(\phi),
$$

and the scalar-field equation of motion is

$$
\ddot{\phi}+3 H \dot{\phi}=-\frac{d V}{d \phi} \text {. }
$$

We solve these coupled differential equations for the scale factor $a(t)$ and scalar field $\phi(t)$ numerically. The solution is insensitive to our choice of initial conditions for $\phi(t)$, as the solution exhibits an attractor behavior if the field begins high enough in the potential [20].

We then turn our attention to the perturbations. We express the power spectrum $\mathcal{P}_{\mathcal{R}}$ of the primordial curvature perturbation with the horizon-crossing approximation $[20,21]$,

$$
\mathcal{P}_{\mathcal{R}}(k)=\left[1-2(2 C+1) \epsilon_{H}+2 C \eta_{H}\right]\left(\frac{H^{2}}{2 \pi|\dot{\phi}|}\right)^{2},
$$

where $C=-2+\ln 2+b \simeq-0.73$, with $b$ the EulerMascheroni constant. The right-hand side is evaluated at $k=a H$, and the Hamilton-Jacobi slow-roll parameters are

$$
\epsilon_{H} \equiv \frac{1}{2} \frac{\dot{\phi}^{2}}{H^{2}}, \quad \eta_{H} \equiv-\frac{\ddot{\phi}}{H \dot{\phi}} .
$$

The next step is to relate a value of $\phi$ to a comoving wave number $k$ that crosses the horizon at that value of $\phi$. To do so, we note that the number of $e$ foldings of inflation between a time $t$ and the end of inflation is

$$
N(t) \equiv \ln \frac{a\left(t_{\text {end }}\right)}{a(t)},
$$

and in terms of $k$, it is

$$
N(k) \simeq 55-\ln \frac{k}{a_{0} H_{0}},
$$

where $a_{0}$ is the scale factor today (which we choose to be $\left.a_{0}=1\right)$, and $H_{0}=(h / 3000) \mathrm{Mpc}^{-1}$ with $h \simeq 0.72$. Note that the uncertainty around the $55 e$ foldings of inflation at horizon crossing is about 5 , and comes mainly from the uncertainty in the reheating process [22].

The distance scale $\lambda$ that exits the horizon varies roughly as the exponential of the change $\delta \phi$ in the inflaton $\phi$. Hence an oscillation in $\phi$ in the inflaton potential should give rise to oscillations in the logarithm of the wave number $k$ in the primordial power spectrum, and thus in the logarithm of the CMB multipole moment $\ell$.

We then calculate the temperature and polarization (TT, $\mathrm{EE}$, and TE) spectra for the model using the CAMB code [23], using the current best-fit parameters for a $\Lambda \mathrm{CDM}$ model from the WMAP 5-year results [18]. We neglect the $B$-mode polarization, as it comes about either through gravitational waves or gravitational lensing and is always small compared with the $E$-mode polarization.
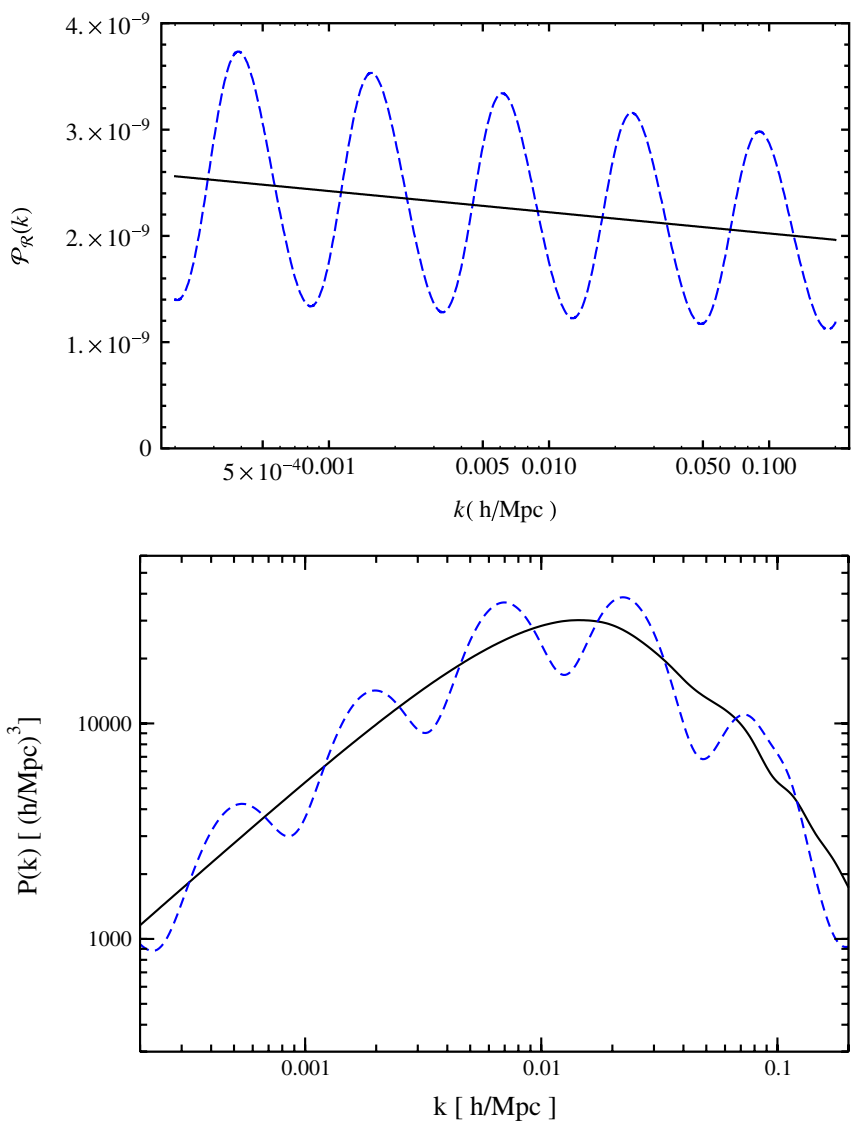

FIG. 1 (color online). Primordial power spectrum (top panel) and matter power spectrum (bottom panel) of the smooth inflaton potential (solid line) and oscillating potential (dashed line). The oscillating-potential parameters are $[\alpha, \beta]=\left[5 \times 10^{-4}, 3 \times\right.$ $\left.10^{-2}\right]$. The amplitude is chosen to be large to clearly show the effect of the oscillations. 
OSCILLATIONS IN THE INFLATON POTENTIAL?

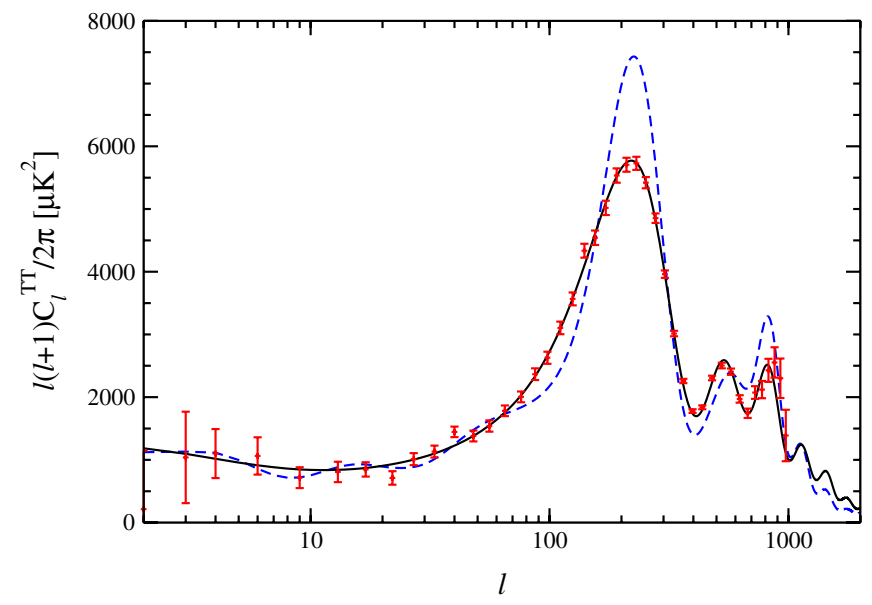

FIG. 2 (color online). The CMB power spectrum corresponding to the models shown in Fig. 1. The WMAP5 data are superimposed [18]. The error bars include both the cosmic variance and instrumental noise.

\section{POWER SPECTRA}

We begin by showing in Fig. 1 the primordial power spectrum, as well as the present matter power spectrum, for the standard smooth potential and for an oscillating potential with parameters chosen to be $[\alpha, \beta]=\left[5 \times 10^{-4}, 3 \times\right.$ $\left.10^{-2}\right]$. The oscillations in the power spectra are nearly sinusoidal in $\log k$ with almost constant amplitude. The corresponding $\mathrm{CMB}$ angular power spectrum, shown in Fig. 2, reflects this behavior. The mapping from the three-dimensional matter power spectrum to the twodimensional $\mathrm{CMB}$ power spectrum slightly smooths the wiggles. In this example, the frequency $\beta$ of the inflationary potential's oscillations has been chosen so that one of the primordial oscillation peaks lines up with the first acoustic peak. However, this coincidence will not be generic.

\section{A SEARCH IN WMAP DATA}

Given the CMB predictions discussed above, it is straightforward to search the existing 5-year CMB data from WMAP for these oscillations, and we have carried out a Markov Chain Monte Carlo (MCMC) analysis to do so. Figure 3 presents the results of this analysis, assuming that all other cosmological parameters are known. It is important to note here that we do not expect strong degeneracies between the primordial oscillations and the acoustic oscillations, because they have different periodicities-in $\ln \ell$ for the former and in $\ell$ itself for the latter. Accordingly, the sensitivity to our oscillations is not affected by keeping other parameters fixed, and nor do we expect polarization data to have a big impact on the constraints (unlike the case of primordial oscillations which are linear in $\ell$, where the phase shift of $180^{\circ}$ in the polarization spectra can break this sort of degeneracy).
PHYSICAL REVIEW D 79, 083503 (2009)

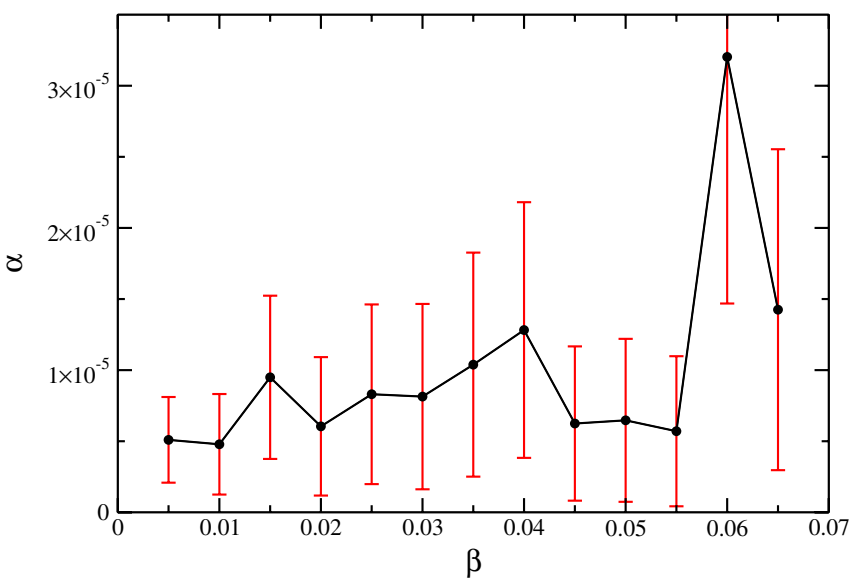

FIG. 3 (color online). The best-fit amplitude $\alpha$ and its standard error, considering WMAP5 data, for our range of frequencies $\beta$.

For each point, we run four Markov chains. The results provide an indication of the best-fit amplitude $\alpha$ as a function of the assumed frequency $\beta$. These amplitudes are comparable to their standard errors, and so we conclude that there is no evidence for oscillations in the WMAP data. Instead, we infer an upper limit $\alpha \lesssim 3 \times 10^{-5}$ from WMAP. We note that a corresponding MCMC analysis has already been performed in Ref. [14] for the WMAP3 data for a similar potential. They find a precision on the amplitude of oscillations of the order of $10^{-5}$ for WMAP3, consistent with our results.

\section{DETECTABILITY}

We now estimate the smallest oscillation amplitude $\alpha$ that will be detectable with future experiments. To do so, we first consider for simplicity the temperature power spectrum only. We suppose that each multipole moment $\ell$ can be measured with a standard error

$$
\sigma_{l}=\sqrt{\frac{2}{(2 l+1) f_{\mathrm{sky}}}}\left(C_{l}+N_{l}\right)
$$

where

$$
N_{l} \equiv\left(\overline{\Delta T}^{2} \theta_{b}^{2}\right) \exp \frac{l^{2} \theta_{b}^{2}}{8 \ln 2}
$$

is the contribution from the detector noise, $\overline{\Delta T}$ is the detector noise per angular-resolution element, and $\theta_{b}$ is the beam width. We then estimate the error on $\alpha$ by [24]

$$
\left(\frac{1}{\sigma_{\alpha}}\right)^{2}=\sum_{l}\left(\frac{\partial C_{l}}{\partial \alpha}\right)^{2} \frac{1}{\sigma_{l}^{2}(\alpha)},
$$

and we choose an amplitude $\alpha=5 \times 10^{-5}$ to compute the $C_{l}$ derivatives. This estimate assumes that all other cosmological parameters are known, and as such, provides an optimistic estimate. However, as argued above, the true value, obtained by marginalizing over all other cosmologi- 
cal parameters, will probably not be too much worse, as there are no strong degeneracies between these oscillations and any other cosmological parameters due to their oscillations being uniform in $\ln \ell$.

In order to improve our results, we then include the polarization and temperature-polarization power spectra as well. In that case, the generalization of the expression for the smallest detectable oscillation takes the form [25]

$$
\left(\frac{1}{\sigma_{\alpha}}\right)^{2}=\sum_{l} \sum_{A, A^{\prime}} \frac{\partial C_{l}^{A}}{\partial \alpha}\left[\Psi^{-1}\right]_{A A^{\prime}} \frac{\partial C_{l}^{A^{\prime}}}{\partial \alpha},
$$

for $A=\mathrm{TT}, \mathrm{EE}$, TE where $\left[\Psi^{-1}\right]_{A A^{\prime}}$ are elements of the inverse of $\Psi$, the covariance matrix; its elements are given in Ref. [25].

In Figs. 4 and 5, we plot the smallest detectable amplitude $\sigma_{\alpha}$, from these analyses, as a function of $\beta$, for WMAP (in Fig. 4 only), Planck, and a hypothetical cosmic-variance limited experiment. For WMAP, we simply consider the measured uncertainties from the 5-year results [18]. For Planck, the forecast is done considering the three most sensitive temperature channels, of specifications similar to the HFI channels of frequency $100 \mathrm{GHz}$, $143 \mathrm{GHz}$, and $217 \mathrm{GHz}$ [2]. The intensity sensitivities (detector noise) of these channels are taken as $6.8 \mu \mathrm{K}$, $6.0 \mu \mathrm{K}$, and $13.1 \mu \mathrm{K}$ respectively, corresponding to the values quoted for two complete sky surveys. These are average sensitivities per pixel, where a pixel is a square whose side is the FWHM extent of the beam. The FWHMs (beam widths) of these channels are given as 9.5 arcmin, 7.1 arcmin, and 5.0 arcmin, respectively. The composite noise spectrum for the three temperature channels is obtained by inverse-variance weighting the noise of individual channels [26,27]. For polarization we take only one channel, the $143 \mathrm{GHz}$ channel, of sensitivity $11.5 \mu \mathrm{K}$, and

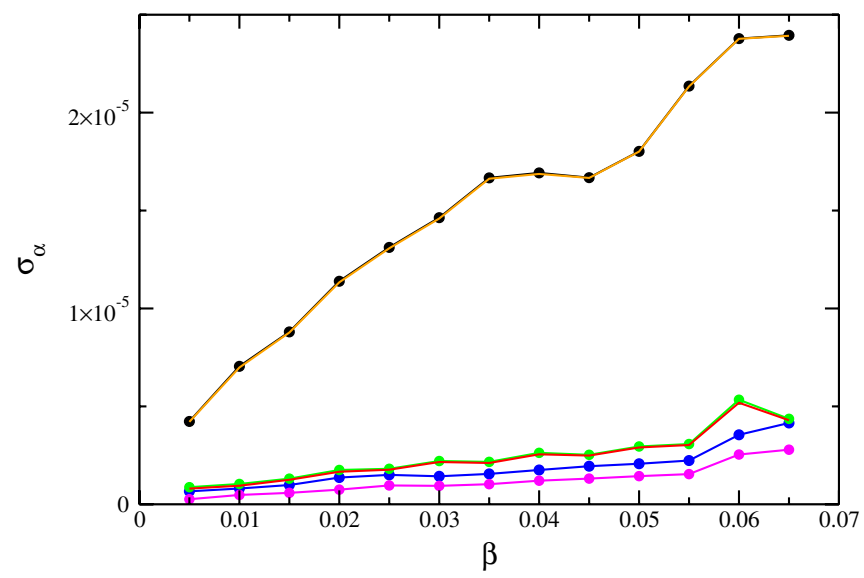

FIG. 4 (color online). From the top to the bottom, by pairs, the smallest detectable amplitude $\sigma_{\alpha}$ as a function of $\beta$, for WMAP, Planck, and a cosmic-variance limited experiment. The upper part of each pair includes the temperature data only, and the lower one the polarization data as well. Note that the temperature and temperature-polarization curves for WMAP and Planck are effectively degenerate and thus appear to be one curve.

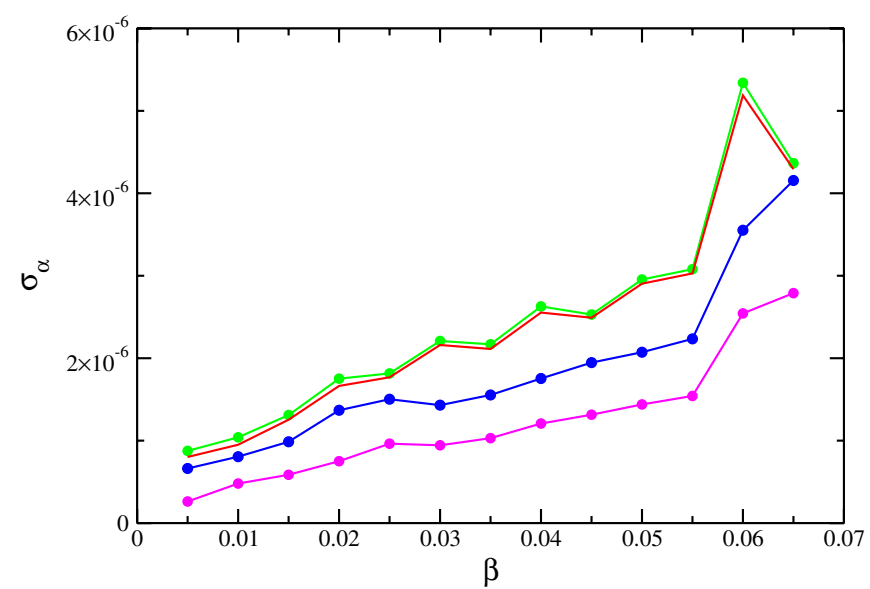

FIG. 5 (color online). Magnification of Fig. 4 for Planck and the cosmic-variance limited experiment.

FWHM 7.1 arcmin. Finally, for the cosmic-variance limited experiment, the fractional sky covered is taken to be 0.8 for all $\ell$, and we use simulated data out to an $\ell_{\max }$ of 2000, as for Planck.

The statistical analysis presented in this section is intended to forecast the uncertainty on parameters measured by future experiments. It is nevertheless reassuring that this forecast, when applied to WMAP, recovers roughly the limits obtained from the detailed MCMC analysis. Figure 4 illustrates the advantage of Planck over WMAP for an oscillation search. While the smallest detectable amplitude is $O\left(10^{-5}\right)$ for WMAP, it is $O\left(10^{-6}\right)$ for Planck. Looking at Planck more closely, in Fig. 5, we find a smooth variation of $\sigma_{\alpha}$ except at some frequencies, such as $\beta=0.03$ and $\beta=0.04$ for instance. These deviations arise from correlations between the acoustic peaks and the primordial peaks, coming from the inflaton potential, in the CMB power spectrum. When varying the frequency, the bumps thus created are aligned differently with the acoustic oscillations. For example, the choice of $\beta=$ 0.03 considered in Fig. 2 is particular as many bumps are well aligned with the acoustic peaks. This effect is however much less important at high frequency, as our results suggest.

The temperature-only and temperature-polarization curves coincide for both WMAP and Planck. This follows because the polarization amplitude is much smaller and neither WMAP nor Planck will measure polarization to the cosmic-variance limit. The contribution to the total signalto-noise from polarization in these experiments is thus small. The degeneracy is broken in the cosmic-variance limited experiment. In this case, the precisions with which the temperature and polarization power spectra can be measured are roughly the same, resulting in roughly a $\sqrt{2}$ improvement to the oscillation sensitivity.

Figures 4 and 5 indicate that a higher frequency of oscillations (i.e. a smaller $\beta$ ) allows a smaller detectable oscillation amplitude. This can be understood by consid- 
ering Eq. (10), whose result is mainly governed by the difference between the chaotic and the oscillating curve, in terms of amplitudes. For a given amplitude of oscillations in the CMB power spectrum, but different frequencies, the difference between these two curves is almost the same (it would be exactly the same if the slope of the chaotic spectrum was constant). However, in our analysis, we are considering a given amplitude $\alpha$ in the inflaton potential itself. When the frequency of oscillations in the inflaton potential increases, so does that in the CMB power spectrum, but the corresponding amplitude in the CMB spectrum also increases (rather than staying constant). As such, for a given $\alpha$, the difference between the chaotic and oscillating curve increases when $\beta$ decreases, and hence $\sigma_{\alpha}$ decreases.

We can now also justify our neglect of the phase $\delta$ introduced in Sec. II. Its effect would simply be to shift the deviations on the smooth curves. In Sec. II, we assumed that $\alpha$ was sufficiently small that inflation was not interrupted, and we can now justify that assumption. The constraint on $\alpha$ from WMAP is already $\alpha \lesssim 3 \times 10^{-5}$. In the case of the most critical values considered in our analysis, being $[\alpha, \beta]=\left[5 \times 10^{-5}, 5 \times 10^{-3}\right]$, we get $\left[\epsilon_{H}^{\max },\left|\dot{\epsilon}_{H}^{\max }\right|\right] \simeq$ $\left[1 \times 10^{-2}, 2 \times 10^{-7}\right]$ and $\left[\left|\eta_{H}^{\max }\right|,\left|\dot{\eta}_{H}^{\max }\right|\right] \simeq\left[2 \times 10^{-1}, 2 \times\right.$ $10^{-4}$, well within the slow-roll regime.

\section{CONCLUSIONS}

The purpose of our paper has been to study whether features in the inflaton potential, beyond the standard slowroll parameterization, can be detected with current and forthcoming CMB experiments. To do so, we have considered the possibility that the inflaton potential varies sinusoidally with the inflaton. A sinusoidal variation of $V(\phi)$ with $\phi$ induces a variation in the matter and CMB power spectra that is sinusoidal in $\log k$ and $\log \ell$, respectively.
Our analysis of the WMAP data indicates that the upper bound on $\alpha$, the oscillation amplitude, is roughly $3 \times$ $10^{-5}$. To derive this bound, we argued that the degeneracy between $\alpha$ and other cosmological parameters is weak, and then assumed in the data analysis that the other parameters were fixed. We anticipate that a more complete analysis, including marginalization over other parameters, will weaken this bound, but only slightly. We then showed that the sensitivity of Planck to the oscillation amplitude will be greater, relative to WMAP, by roughly an order of magnitude. For both Planck and WMAP, the constraint comes primarily from the temperature. Planck might be improved upon slightly by a cosmic-variance limited experiment. The advantage of such an experiment is that the additional information in the polarization may then be fully capitalized upon.

On the theory side, we used the horizon-crossing approximation to calculate the power spectrum. The work of Ref. [17] suggests that numerical integration of the perturbation equations will result in a slight suppression of the oscillation amplitude, relative to that we have obtained, but certainly by no more than a factor of 2 . If so, then our bounds may be accordingly weakened. We leave investigation of these data-analysis and theory questions for future research.

\section{ACKNOWLEDGMENTS}

C. P. acknowledges the hospitality of the Moore Center for Theoretical Cosmology and Physics at Caltech. C. P. was supported in part by the Swiss Sunburst Fund and the Royal Astronomical Society; M. K. was supported by the DOE under Grant No. DE-FG03-92-ER40701 and the Gordon and Betty Moore Foundation; and A.R. L. was supported by STFC (UK).
[1] P. de Bernardis et al. (Boomerang Collaboration), Nature (London) 404, 955 (2000); A. D. Miller et al., Astrophys. J. 524, L1 (1999); S. Hanany et al., Astrophys. J. 545, L5 (2000); N. W. Halverson et al., Astrophys. J. 568, 38 (2002); B. S. Mason et al., Astrophys. J. 591, 540 (2003); A. Benoit et al. (The Archeops Collaboration), Astron. Astrophys. 399, L25 (2003); J. H. Goldstein et al., Astrophys. J. 599, 773 (2003); D. N. Spergel et al. (WMAP Collaboration), Astrophys. J. Suppl. Ser. 148, 175 (2003); D. N. Spergel et al. (WMAP Collaboration), Astrophys. J. Suppl. Ser. 170, 377 (2007).

[2] Planck Collaboration, arXiv:astro-ph/0604069.

[3] J. A. Adams, G. G. Ross, and S. Sarkar, Nucl. Phys. B503, 405 (1997).

[4] J. A. Adams, B. Cresswell, and R. Easther, Phys. Rev. D 64, 123514 (2001); C. P. Burgess, J. M. Cline, F. Lemieux, and R. Holman, J. High Energy Phys. 02 (2003), 048; H. V. Peiris et al. (WMAP Collaboration), Astrophys. J. Suppl. Ser. 148, 213 (2003); C. R. Contaldi, M. Peloso, L. Kofman, and A. Linde, J. Cosmol. Astropart. Phys. 07 (2003), 002.

[5] R. H. Brandenberger and J. Martin, Mod. Phys. Lett. A 16, 999 (2001); J. Martin and R. Brandenberger, Phys. Rev. D 68, 063513 (2003).

[6] R. Easther, B. R. Greene, W. H. Kinney, and G. Shiu, Phys. Rev. D 66, 023518 (2002).

[7] J. Martin and C. Ringeval, Phys. Rev. D 69, 083515 (2004); 69, 127303 (2004); J. Cosmol. Astropart. Phys. 01 (2005) 007.

[8] T. Okamoto and E. A. Lim, Phys. Rev. D 69, 083519 (2004); R. Easther, W. H. Kinney, and H. Peiris, J. Cosmol. Astropart. Phys. 05 (2005) 009. 
[9] A. A. Starobinsky, Pis'ma Zh. Eksp. Teor. Fiz. 55, 477 (1992) [JETP Lett. 55, 489 (1992)]; N. Kaloper and M. Kaplinghat, Phys. Rev. D 68, 123522 (2003); M. Kawasaki, F. Takahashi, and T. Takahashi, Phys. Lett. B 605, 223 (2005); A. Ashoorioon and A. Krause, arXiv: hep-th/0607001; C. Pitrou, T. S. Pereira, and J.P. Uzan, J. Cosmol. Astropart. Phys. 04 (2008) 004.

[10] A. Shafieloo and T. Souradeep, Phys. Rev. D 70, 043523 (2004); A. Shafieloo, T. Souradeep, P. Manimaran, P. K. Panigrahi, and R. Rangarajan, Phys. Rev. D 75, 123502 (2007); A. Shafieloo and T. Souradeep, Phys. Rev. D 78, 023511 (2008).

[11] N. Kogo, M. Matsumiya, M. Sasaki, and J. Yokoyama, Astrophys. J. 607, 32 (2004); N. Kogo, M. Sasaki, and J. Yokoyama, Phys. Rev. D 70, 103001 (2004).

[12] C. Sealfon, L. Verde, and R. Jimenez, Phys. Rev. D 72, 103520 (2005).

[13] L. Verde and H. V. Peiris, J. Cosmol. Astropart. Phys. 07 (2008) 009.

[14] L. Covi, J. Hamann, A. Melchiorri, A. Slosar, and I. Sorbera, Phys. Rev. D 74, 083509 (2006); J. Hamann, L. Covi, A. Melchiorri, and A. Slosar, Phys. Rev. D 76, 023503 (2007).

[15] P. Hunt and S. Sarkar, Phys. Rev. D 70, 103518 (2004); 76, 123504 (2007).

[16] X. Chen, R. Easther, and E. A. Lim, J. Cosmol. Astropart.
Phys. 04 (2008), 010.

[17] X. Wang, B. Feng, M. Li, X. L. Chen, and X. Zhang, Int. J. Mod. Phys. D 14, 1347 (2005).

[18] E. Komatsu et al. (WMAP Collaboration), Astrophys. J. Suppl. Ser. 180, 330 (2009); M. R. Nolta et al. (WMAP Collaboration), Astrophys. J. Suppl. Ser. 180, 296 (2009).

[19] R. Bean, X. Chen, G. Hailu, S.-H. H. Tye, and J Xu, J. Cosmol. Astropart. Phys. 03 (2008), 026.

[20] A. R. Liddle and D. H. Lyth, Cosmological Inflation and Large-Scale Structure (Cambridge University Press, Cambridge, England, 2000).

[21] E. D. Stewart and D. H. Lyth, Phys. Lett. B 302, 171 (1993).

[22] A. R. Liddle and S. M. Leach, Phys. Rev. D 68, 103503 (2003).

[23] http://camb.info/.

[24] G. Jungman, M. Kamionkowski, A. Kosowsky, and D. N. Spergel, Phys. Rev. D 54, 1332 (1996); G. Jungman, M. Kamionkowski, A. Kosowsky, and D. N. Spergel, Phys. Rev. Lett. 76, 1007 (1996).

[25] M. Kamionkowski, A. Kosowsky, and A. Stebbins, Phys. Rev. D 55, 7368 (1997); M. Zaldarriaga and U. Seljak, Phys. Rev. D 55, 1830 (1997).

[26] L. Knox, Phys. Rev. D 52, 4307 (1995).

[27] A. R. Cooray and W. Hu, Astrophys. J. 534, 533 (2000). 